University of Nebraska - Lincoln

DigitalCommons@University of Nebraska - Lincoln

Sociology Department, Faculty Publications

Sociology, Department of

2005

Family Economic Pressure and Adolescent Suicidal Ideation: Application of the Family Stress Model

Kevin A. Yoder

University of North Texas, yoder@scs.unt.edu

Dan R. Hoyt

University of Nebraska-Lincoln, dhoyt2@unl.edu

Follow this and additional works at: https://digitalcommons.unl.edu/sociologyfacpub

Yoder, Kevin A. and Hoyt, Dan R., "Family Economic Pressure and Adolescent Suicidal Ideation: Application of the Family Stress Model" (2005). Sociology Department, Faculty Publications. 212. https://digitalcommons.unl.edu/sociologyfacpub/212

This Article is brought to you for free and open access by the Sociology, Department of at DigitalCommons@University of Nebraska - Lincoln. It has been accepted for inclusion in Sociology Department, Faculty Publications by an authorized administrator of DigitalCommons@University of Nebraska - Lincoln. 
Published in Suicide and Life-Threatening Behavior 35:3 (June 2005), pp. 251-264; doi: 10.1521/suli.2005.35.3.251

Copyright (C) 2005 The American Association for Suicidology. Published by John Wiley \& Sons. Used by permission.

Submitted June 23, 2003; accepted August 30, 2004; published online June 1, 2011.

The Mississippi Delta Study was supported by the National Institute of Mental Health (MH48165) and the U.S. Department of Agriculture; Dan R. Hoyt, Principal Investigator.

\title{
Family Economic Pressure and Adolescent Suicidal Ideation: Application of the Family Stress Model
}

\author{
Kevin A. Yoder \\ University of North Texas \\ (Corresponding author: yoder@scs.unt.edu ) \\ Dan R. Hoyt \\ University of Nebraska-Lincoln
}

\begin{abstract}
This study used a sample of 501 families from the Mississippi Delta region to examine the feasibility of the Family Stress Model for understanding adolescent suicidal ideation. The results indicated that family economic pressure was related to parental depressive symptoms, which, in turn, was related to parental hostile behavior and physical abuse. These parental behaviors were related to adolescent self-esteem and depressive symptoms, which, in turn, were related to adolescent suicidal ideation. Therefore, family economic pressure and parental depressive symptoms and behaviors were indirectly related to adolescent suicidal ideation. The results indicate the applicability of the Family Stress Model for understanding adolescent suicidal ideation.
\end{abstract}

S uicidal ideation is relatively common among adolescents in the general population, and it is highly correlated with suicide attempts (Lewinsohn, Rohde, \& Seeley, 1996). This suggests that suicide attempts and completed suicide might be averted, in part, by recognizing and treating suicidal ideation. It is thus important to determine characteristics of adolescents that are related to suicidal ideation, and many studies of adolescents in the general population have examined correlates of suicidal ideation.

Adolescent characteristics such as self-esteem and depressive symptoms (e.g., Overholser, Adams, Lehnert, \& Brinkman, 1995) as well as family characteristics such as parental hostility or phys- ical abuse (e.g., Bensley, Van Eenwyk, Spieker, \& Schoder, 1999; Shagle \& Barber, 1993), parental depressive symptoms (e.g., Garber, Little, Hilsman, \& Weaver, 1998), and socioeconomic status (e.g., Dubow, Kausch, Blum, Reed, \& Bush, 1989) have emerged as important correlates of suicidal ideation among adolescents in the general population. Nonetheless, the mechanisms by which these variables relate to adolescent suicidal ideation remain poorly specified, and the present study examines the feasibility of the Family Stress Model (Conger \& Elder, 1994) for understanding these relationships in a sample of 501 families from the Mississippi Delta region. 


\section{Theoretical Model and Prior Research}

Conger and Elder (1994) proposed the Family Stress Model (FSM) to understand the effects of family economic pressure on parents and adolescents in rural Iowa farm communities. The FSM indicates that family economic pressure (financial problems) influences parental emotional distress that, in turn, impacts their parenting behaviors, which themselves affect adolescent psychological well-being. Thus, parenting behaviors mediate the relationships of family economic stress and parental emotional distress with adolescent psychological well-being (Conger \& Elder, 1994).

The present study extends the FSM by including adolescent suicidal ideation. The theoretical model to be tested is illustrated in Figure 1 (the hypothesized paths are labeled with letters and the expected direction in parentheses). First, it is hypothesized that family economic pressure will be positively related to parental depressive symptoms (Path A). This hypothesis is justified by the stress process paradigm (Pearlin, 1989) in which stressful life events (economic pressure) influence mental health outcomes, and previous research supports this hypothesis (Barrera et al., 2002). Second, it is hypothesized that parental depressive symptoms will be positively related to parental hostile behavior toward, and physical abuse of, the adolescent (Paths B and C). Downey and Coyne (1990) note that maternal depression results in hostile and rejecting behavior toward their children, and researchers have supported the hypothesized relationships (e.g., Chaffin, Kelleher, \& Hollenberg, 1996; Conger et al., 1991).

Third, it is hypothesized that parental hostile behavior toward the adolescent will be positively related to parental physical abuse of the adolescent (Path D). This association may reflect an extremely coercive and hostile parenting style, or it may reflect antecedent parental experiences that are associated with hostile and abusive parenting (Crittenden, 1998). Fourth, it is hypothesized that parental hostile behavior and physical abuse will be negatively related to adolescent self-esteem (Paths E and $\mathrm{H}$ ) and positively related to adolescent depressive symptoms (Paths F and I) and suicidal ide- ation (Paths G and J). Hostile and abusive parental practices might convey the message that the adolescent is unworthy, which in turn, could be internalized by the adolescent (Gecas \& Schwalbe, 1986). Self-blame for these parental behaviors could result in depressive symptoms and suicidal thoughts (Simons, Johnson, \& Conger, 1994; Wagner, Silverman, \& Martin, 2003). Previous research has supported the hypothesized relationships between parental support and adolescent self-esteem (e.g., Whitbeck et al., 1991), parental hostile behavior and adolescent depressive symptoms (Conger, Ge, Elder, Lorenz, \& Simons, 1994), parent-child conflict and adolescent suicidal ideation (Shagle \& Barber, 1993), and parental physical abuse with children's or adolescent's self-esteem, depression, and suicidal ideation (e.g., Allen \& Tarnowski, 1989; Bensley et al., 1999).

Fifth, it is hypothesized that adolescent self-esteem will be negatively related to adolescent depressive symptoms and suicidal ideation (Paths $\mathrm{K}$ and L). Self-esteem theory posits that people have a "self-maintenance motive" to view themselves in a positive light, and failing this, they may react by becoming depressed, feeling hopeless about the future, and believing that life is not worth living (Overholser et al., 1995; Rosenberg, Schooler, \& Schoenbach, 1989). Researchers have found strong relationships of self-esteem with depressive symptoms (e.g., Allgood-Merten, Lewinsohn, \& Hops, 1990) and suicidal ideation (e.g., Overholser et al., 1995) among adolescents.

Sixth, it is hypothesized that adolescent depressive symptoms will be positively related to suicidal ideation. Beck's cognitive theory of depression indicates that people who are depressed might believe that they deserve not to live, and as a result, they are prone to think about suicide (Beck, 1976). Researchers have consistently found a strong relationship between depression and suicidal ideation among adolescents (e.g., Overholser et al., 1995).

Finally, it is hypothesized that family economic pressure will be indirectly related to parenting behaviors (via parental depressive symptoms) and adolescent outcomes (via parental depressive symptoms and parenting behaviors) and that parental depressive symptoms will be indirectly re- 


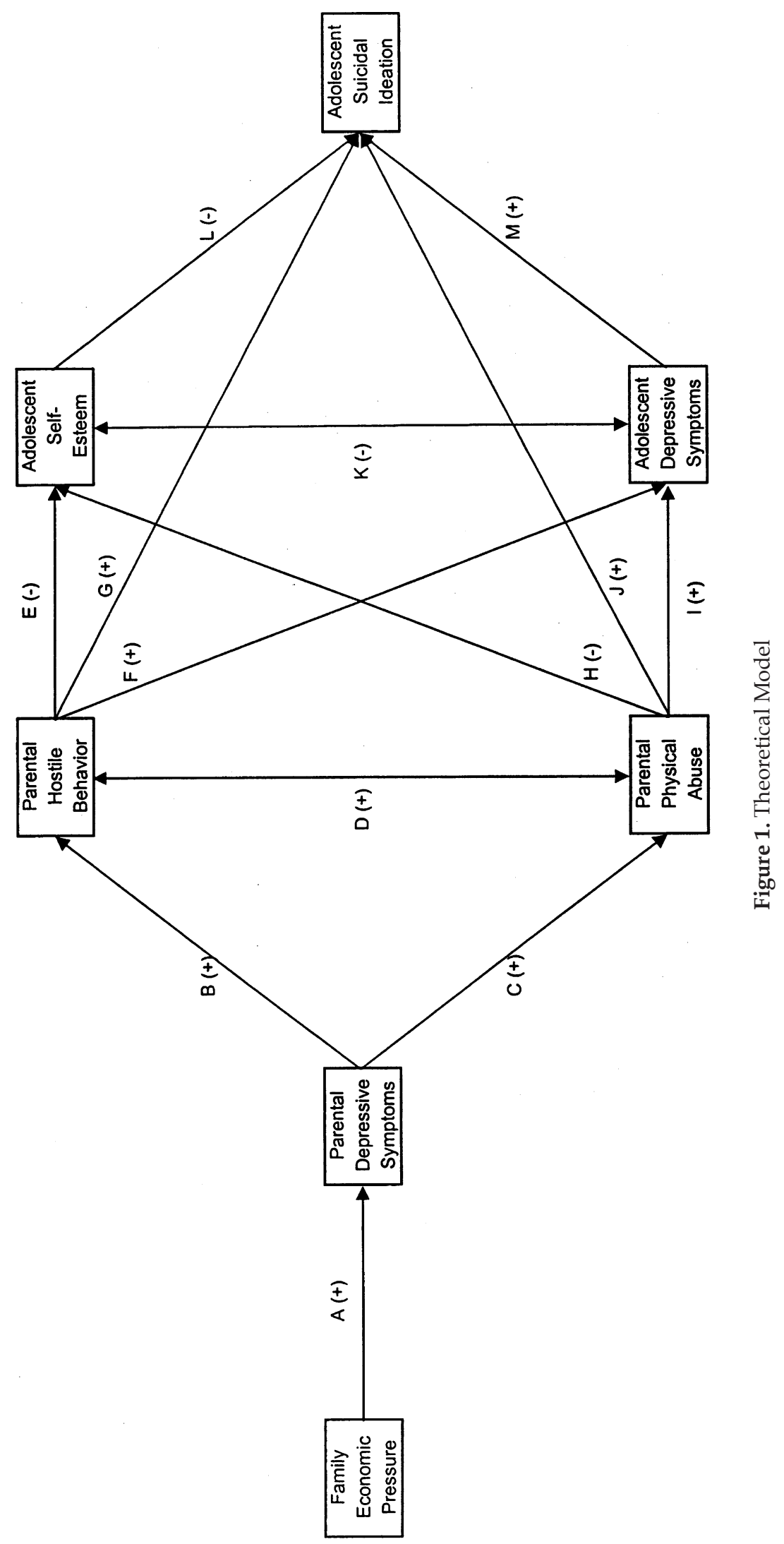


lated to adolescent outcomes (via parenting behaviors). These indirect effects are consistent with the FSM and are supported by previous research. Several studies have found an indirect relationship between family economic pressure and adolescent internalizing symptoms via parental depression and parental hostility or support (e.g., Conger et al., 1994). Garber and colleagues (1998) found an indirect relationship between maternal depression and adolescent suicidal symptoms via family functioning, and Shagle and Barber (1993) reported an indirect relationship between parentchild conflict and adolescent suicidal ideation via adolescent self-esteem. Finally, Kandel, Raveis, and Davies (1991) found an indirect relationship between adolescent closeness to parents and adolescent suicidal ideation via adolescent depressive symptoms.

\section{Method}

\section{Sample}

Data for the Mississippi Delta Study were collected to examine economic stress, mental health, and substance use among families in the lower Mississippi Delta region of the United States. People living in this region are among the most economically deprived in the country, as evidenced by relatively high rates of poverty and low per-capita income (Saunders, 1992). This study thus provides a unique opportunity to examine mental health problems in a relatively disadvantaged sample of families.

Families were recruited from a stratified sample of rural counties and parishes in Mississippi, Arkansas, and Louisiana that were adjacent to the Mississippi River, had a population that was at least 35\% African American, and had a population poverty rate of at least $20 \%$. Counties in Tennessee and in the northern third of Arkansas were excluded because they contained urban centers or had too few African American residents. A total of 18 counties or parishes, which were grouped into six local labor market areas that represented a com- mon economic base, were randomly selected for the final sample.

A sample of 501 families, which consisted of at least one adult who was a parent or guardian of a youth aged 13 to 17 years, was recruited for the study. Households were initially identified by using a list-assisted random digit dialing sample, and households with known addresses were then mailed a letter describing the study. Potential participants were telephoned, and eligibility was assessed. If the family met the eligibility requirements, the interviewer then determined the number of eligible parents or guardians in the household and randomly selected one of those parents or guardians to participate in the study. The selected parent or guardian gave verbal consent to be interviewed and permission to interview the target youth.

If the parent or guardian refused to be interviewed or refused to give consent to interview the target youth, the household was classified as a refusal. Nearly 7 of every 10 parents (69.8\%) agreed to be interviewed and gave consent to interview the target youth. Interviews with parents or guardians lasted an average of 23 minutes, and of those interviewed, $67 \%$ were mothers, $31 \%$ were fathers, and the remaining $2 \%$ were other female guardians of the target youth. Upon completing the interview with the parent or guardian, the interviewer requested permission to interview the target youth. If the target youth was not available at the time, the interviewer arranged for a time to call back. When the target youth was contacted, he or she was read an assent protocol and was given an opportunity to refuse to participate; the target youth was interviewed when both parental/guardian consent and youth assent were received. Most of the target youths (94.6\%) agreed to participate, and interviews lasted an average of 22 minutes.

The questionnaires and survey protocols were approved by the Iowa State University Institutional Review Board. The Board approved the modified informed consent protocol described above, and the Board also approved the protocols for interviewer handling of signs of discomfort or reports of abuse. Interviewers were provided local contact 
and referral numbers for the areas that were being called. No adverse events were reported in the conducting of this survey.

Of the 501 adolescents who participated in the study, over half were female $(52.3 \%)$. Nearly $75 \%$ identified themselves as non-Hispanic White; $23.6 \%$ as African American; $1 \%$ as Asian/Pacific Islander, Hispanic, or bi- or multi-racial; and $0.6 \%$ did not indicate their race/ethnicity. The youths ranged in age from 12 to 18 years $(M=14.94, S D$ $=1.43$ ). The majority of households had two parents $(80.2 \%)$, and the remaining households had one parent who had separated or divorced (8.6\%), had been widowed $(2.4 \%)$, or had never married $(2.4 \%)$. Nearly $16 \%$ of households had incomes of $\$ 25,000$ or below; $30 \%$ had incomes between $\$ 25,000$ and $\$ 45,000 ; 31 \%$ had incomes between $\$ 45,000$ and $\$ 75,000 ; 14 \%$ had incomes of $\$ 75,000$ or more; and $9 \%$ did not provide income information.

\section{Measures}

Three control variables were used in the analyses: adolescent gender $(0=$ female; $1=$ male $)$; adolescent race $(0=$ White, non-Hispanic; $1=$ non-White $)$ and adolescent age (in years).

Family economic pressure was measured with six items adapted from Conger and colleagues (1992). Parents rated on a scale from 1 (strongly agree) to 4 (strongly disagree) the extent to which their income catches up with expenses; they have difficulty paying bills; they have money left at the end of the month; and they have enough money for transportation, food, or medical care. The items were coded and averaged so that higher values of the scale indicated more economic pressure, and Cronbach's a for the scale was 0.82 .

Parental depressive symptoms consisted of the 20 items in the Center for Epidemiologic Studies Depression scale (CES-D; Radloff, 1977). Parents reported on a scale from 1 (none of the time) to 3 (most of the time) how often they experienced depressive symptoms in the week prior to the interview. The items were coded and averaged so that higher values of the scale indicated more depres- sive symptoms. Researchers have provided evidence for satisfactory validity and reliability of scores on the CES-D in adult samples (Radloff, 1977), and in this study, Cronbach's a for the scale was 0.88 .

Parental hostile behavior toward the adolescent contained six items adapted from McGruder, Lorenz, Hoyt, Ge, and Montague (1992). Adolescents rated on a scale from 1 (never) to 5 (always) how often their parent(s) expressed anger toward them, blamed them for their parents' problems, or expressed unhappiness with their actions. The items were coded and averaged so that higher values indicated more hostile behavior, and Cronbach's a for the scale was 0.70 .

Parental physical abuse was measured with five items adapted from the Conflict Tactics Scale (CTS; Straus, 1979). Adolescents rated on a scale from 0 (never) to 3 (many times) how often their parent(s) or adult relative(s) threw something at them in anger; pushed, shoved, or grabbed them in anger; slapped them in the face; hit them with some object; or beat them up. The items were coded and averaged so that higher values of the scale indicated more physical abuse. Researchers have provided evidence for satisfactory validity and reliability of scores on the CTS in other samples (Straus, 1979), and in this study, Cronbach's a for the scale was 0.67 .

Adolescent self-esteem consisted of the ten items in the Rosenberg Self-Esteem Scale (Rosenberg, 1965), and response categories on individual items ranged from 1 (strongly agree) to 5 (strongly disagree). The items were coded and averaged so that higher values of the scale indicated higher self-esteem. Researchers have provided evidence for satisfactory validity and reliability of scores on this scale in other adolescent samples (Rosenberg, 1965), and in this study, Cronbach's a for the scale was 0.78 .

Adolescent depressive symptoms contained five items adapted from the Tri-Ethnic Depression Scale (TEDS; Oetting, Swaim, Edwards, \& Beauvais, 1989). Adolescents rated on a scale from 0 (none of the time) to 2 (most of the time) how often during the week prior to the interview that they had felt unhappy, lonesome, down or low, depressed, or sad. 
The items were coded and averaged so that higher values indicated more depressive symptoms. Researchers have provided evidence for satisfactory validity and reliability of scores on the TEDS in other adolescent samples (Oetting et al., 1989), and in this study, Cronbach's a for the scale was 0.76 .

Finally, adolescent suicidal ideation consisted of two items. Adolescents were asked to indicate $(1=$ yes, $2=n o)$ whether, in the 12 months prior to the interview, they had seriously thought about committing suicide or made plans for committing suicide. A small percentage of adolescents indicated that they had seriously thought about $(7.2 \%)$ or made plans for $(2.4 \%)$ committing suicide. As a result, the variable was constructed as a dichotomous variable $(0=$ no suicidal ideation, $1=$ seriously thought about and/or made plans for committing suicide).

\section{Analytic Strategy}

A simple person-mean substitution method was used to handle missing values within multi-item scales; the mean value of the remaining items was imputed for the missing items as long as at least half of the items had legitimate values. Missing value substitution was not used for the single-item variables. In addition, four of the multi-item scales were highly positively skewed (parental hostile behavior, parental physical abuse, parental depressive symptoms, and adolescent depressive symptoms), and the square root of the scales were used in the analyses.

Initial analyses involved comparing the study variables on adolescent gender, race, age (dichotomized into younger [ages 12-15] and older [ages 16-18]), and family economic pressure (dichotomized into low [at or below the median] and high [above the median]) using independent sample $t$ tests (for continuous variables) and chi-square tests (for dichotomous variables). Pearson correlation coefficients were also computed and examined for preliminary evidence for the theoretical model.

The theoretical model in Figure 1 was estimated as a structural equation model with observed variables using the Weighted Least Squares procedure in LISREL 8 (Jöreskog \& Sörbom, 2001). When dependent variables are ordinal or dichotomous (as with suicidal ideation here), the WLS procedure provides better estimates of standard errors (Jöreskog \& Sörbom, 2001). The analyses used Pearson and biserial correlations ${ }^{1}$ and a bootstrap estimate of the weight matrix. ${ }^{2}$ The fit of the theoretical model was assessed using the chisquare statistic and descriptive goodness-of-fit indices (Schermelleh-Engel, Moosbrugger, \& Müller, 2003).

\section{Results}

\section{Missing Cases and Initial Analyses}

Twenty-nine parents did not complete the CES$\mathrm{D}$ items, and three adolescents did not indicate

1. A biserial correlation is an estimate of the correlation between a normally distributed variable that presumably underlies a dichotomous variable and another normally distributed variable. Biserial correlations were used for the dichotomous dependent variable (adolescent suicidal ideation) but not for the two dichotomous independent variables (adolescent gender and adolescent race) because distributional assumptions are not required for independent variables in structural equation modeling (see Bollen, 1989, for a discussion of this issue).

2. The weight matrix can be poorly estimated for small sample sizes, but this can be remedied by using a bootstrap procedure (Yung \& Bentler, 1994). The bootstrap procedure involves random sampling with replacement from the observed data, and as such, different bootstrap samples can yield different results. The bootstrap estimate of the weight matrix was generated using 500 samples with a sampling fraction of 100 . The procedure was repeated several times using different numbers of samples (200,500, and 1000) and different sampling fractions (50 and 100), and the results did not vary much from those reported here. 
their race. Thus, after listwise deletion of those missing cases, 469 remained for the analyses. Adolescents who were included in the analyses, versus those who were excluded, were significantly younger (by half a year), experienced more depressive symptoms, and were about two times more likely to be White than non-White. The included and excluded cases did not differ significantly on the remaining study variables.

Table 1 presents comparisons of the study variables on adolescent gender, race, age, and family economic pressure. Females experienced, on average, more depressive symptoms than did males (Ms of 0.62 and 0.54 ). When compared to White adolescents, non-White adolescents had, on average, parents with more depressive symptoms (Ms of 0.65 and 0.54 ), less hostile parental behavior (Ms of 0.88 and 1.01), and higher self-esteem (Ms of 4.14 and 3.97). Older adolescents, when compared to younger adolescents, on average experienced less physical abuse (Ms of 0.16 and 0.24 ) and had higher self-esteem (Ms of 4.08 and 3.98). Finally, parents experienced more depressive symptoms, on average, when family economic pressure was high versus low (Ms of 0.68 and 0.48).

Table 2 contains correlation coefficients and descriptive statistics for the study variables. The correlations provide preliminary support for the theoretical model (Figure 1); all of the hypothesized relationships are statistically significant.

\section{Structural Equation Model Results}

Standardized coefficient estimates $(\beta)$ are provided in Figure 2 and the upper half of Table 3, and indirect relationships $\left(\beta_{\mathrm{ie}}\right)$ and coefficients o determination $\left(R^{2} \mathrm{~s}\right)$ are presented in the lower half of Table 3. Although the chi-square statistic does not indicate a good-fitting model, $x^{2}(8)=25.59, p$ $=0.001$, the descriptive goodness-of-fit indices indicate an acceptable to good fit (RMSEA $=0.069$, $\mathrm{NFI}=1.00, \mathrm{NNFI}=0.99, \mathrm{CFI}=1.00, \mathrm{SRMR}=0.032$, GFI $=1.00$, and AGFI $=0.99$; Schermelleh-Engel et al., 2003). ${ }^{3}$ The coefficients of determination range from 0.03 (for parental physical abuse) to 0.49 (for adolescent suicidal ideation).

With the exception of two paths, the theoretical model was supported. Family economic pressure is positively related to parental depressive symptoms $(\beta=0.24)$, and it is indirectly related (in the expected directions) to the remaining variables: parental hostile behavior $\left(\beta_{\text {ie }}=0.04\right)$ and physical abuse $\left(\beta_{\text {ie }}=0.03\right)$ via parental depressive symptoms as well as adolescent self-esteem $\left(\beta_{\text {ie }}=-0.02\right)$, depressive symptoms $\left(\beta_{\text {ie }}=0.02\right)$, and suicidal ideation $\left(\beta_{\mathrm{ie}}=0.02\right)$ via parental depressive symptoms and parenting behaviors. Moreover, parental depressive symptoms is positively related to parental hostile behavior $(\beta=0.18)$ and physical abuse $(\beta=0.13)$ and indirectly related (in the expected directions) to adolescent self-esteem $\left(\beta_{\mathrm{ie}}=-0.06\right)$, depressive symptoms $\left(\beta_{\text {ie }}=0.06\right)$, and suicidal ideation $\left(\beta_{\mathrm{ie}}=0.09\right)$.

As expected, parental hostile behavior is related (in the expected directions) to physical abuse ( $\beta=$ $0.31)$, adolescent self-esteem $(\beta=-0.25)$, depressive symptoms $(\beta=0.32)$, and suicidal ideation, both directly $(\beta=0.19)$ and indirectly $\left(\beta_{\mathrm{ie}}=0.19\right)$. Unexpectedly, however, parental physical abuse is not significantly related to adolescent depressive symptoms and only approaches significance in its direct relationship with suicidal ideation. Nonetheless, parental physical abuse is negatively related to adolescent self-esteem $(\beta=-0.14)$ and positively indirectly related to suicidal ideation $\left(\beta_{\mathrm{ie}}=0.06\right)$. Finally, adolescent self-esteem is negatively related

3. The modification index for the path between family economic pressure and parental hostile behavior was 11.99, and estimating this path would have resulted in a better-fitting model. However, the coefficient would have been -0.13 , which is not theoretically reasonable. Post-hoc model modification is generally not recommended without theoretical justification (Kaplan, 1989); therefore, the model was not changed. 


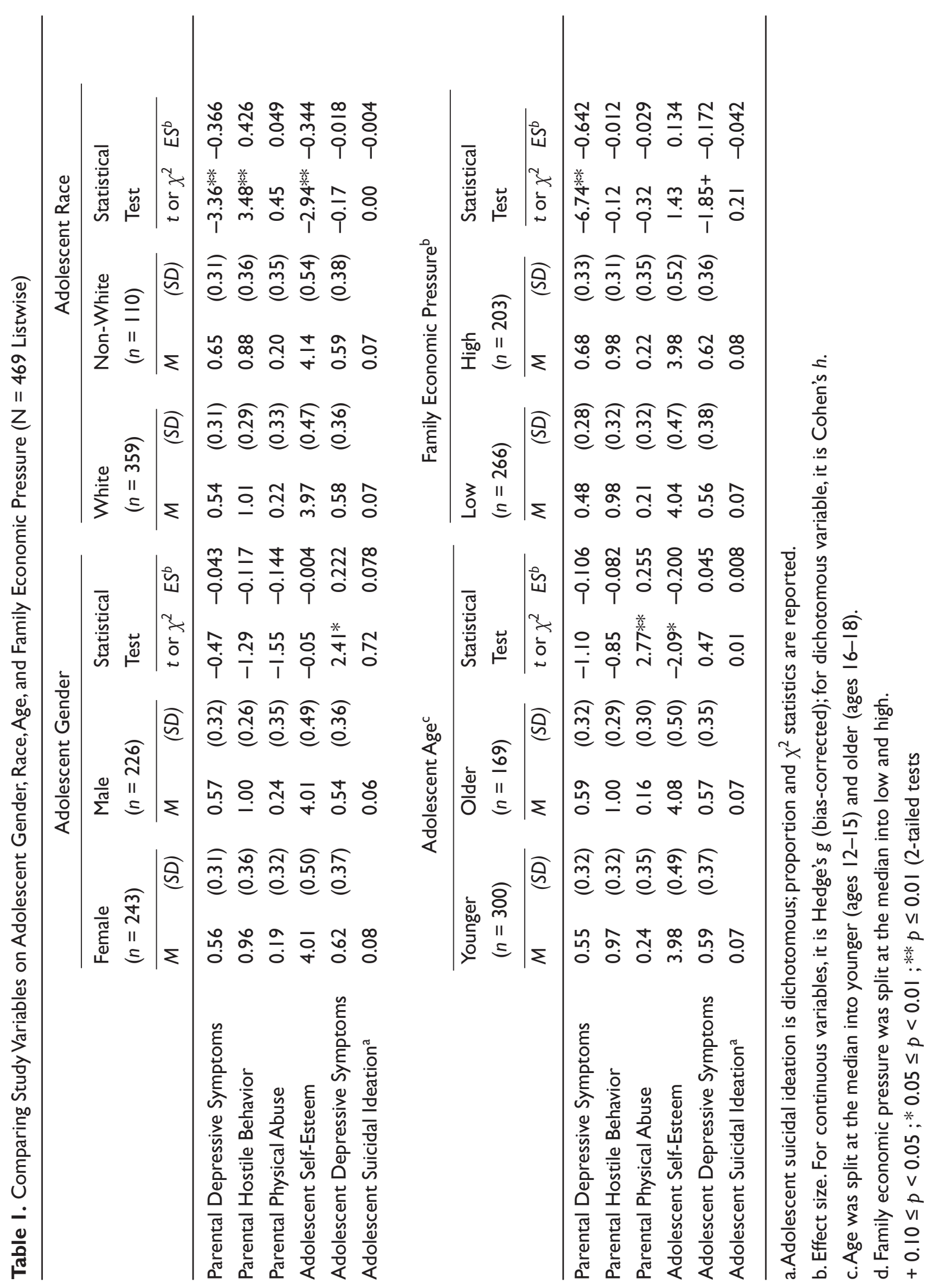




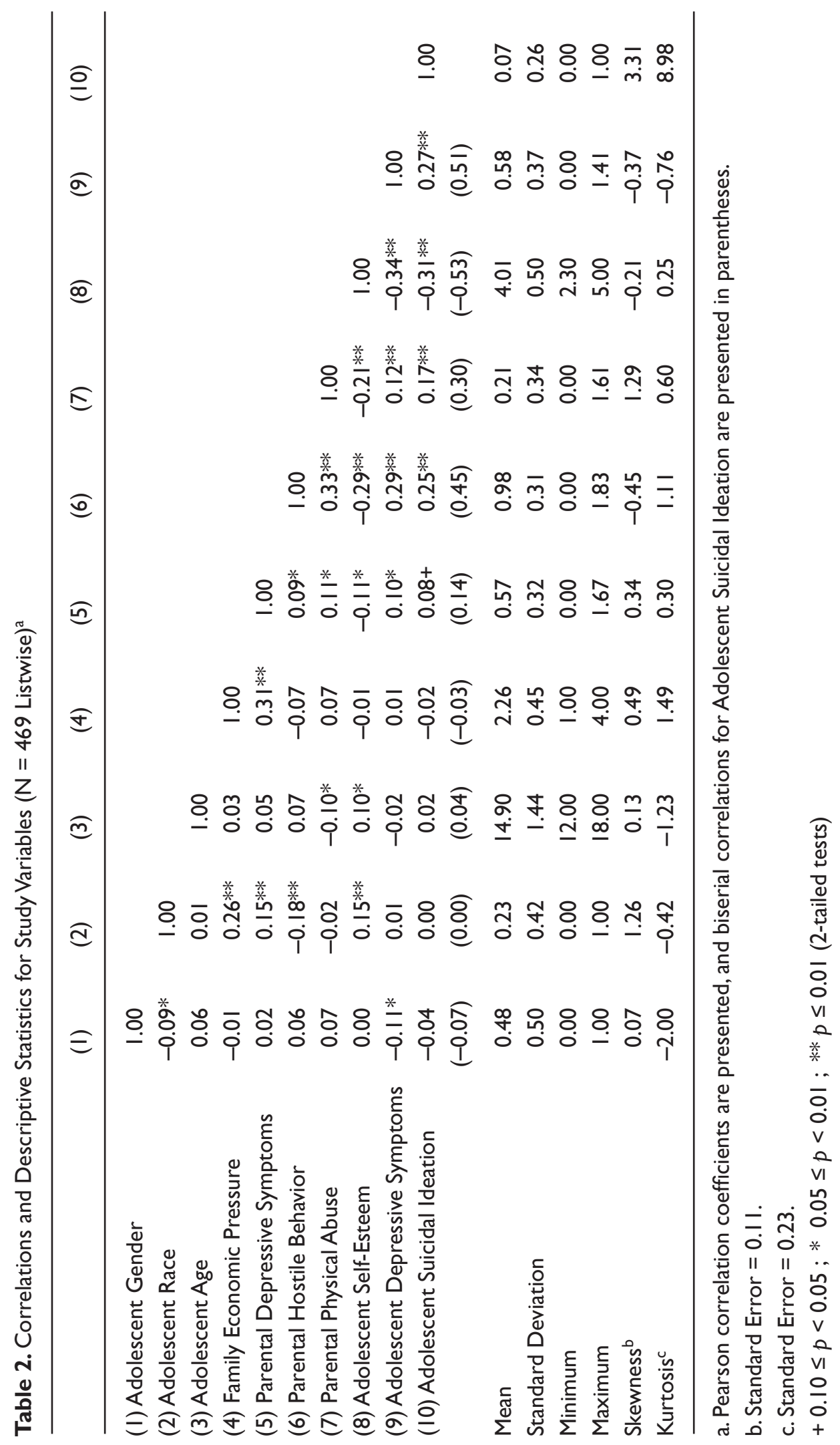




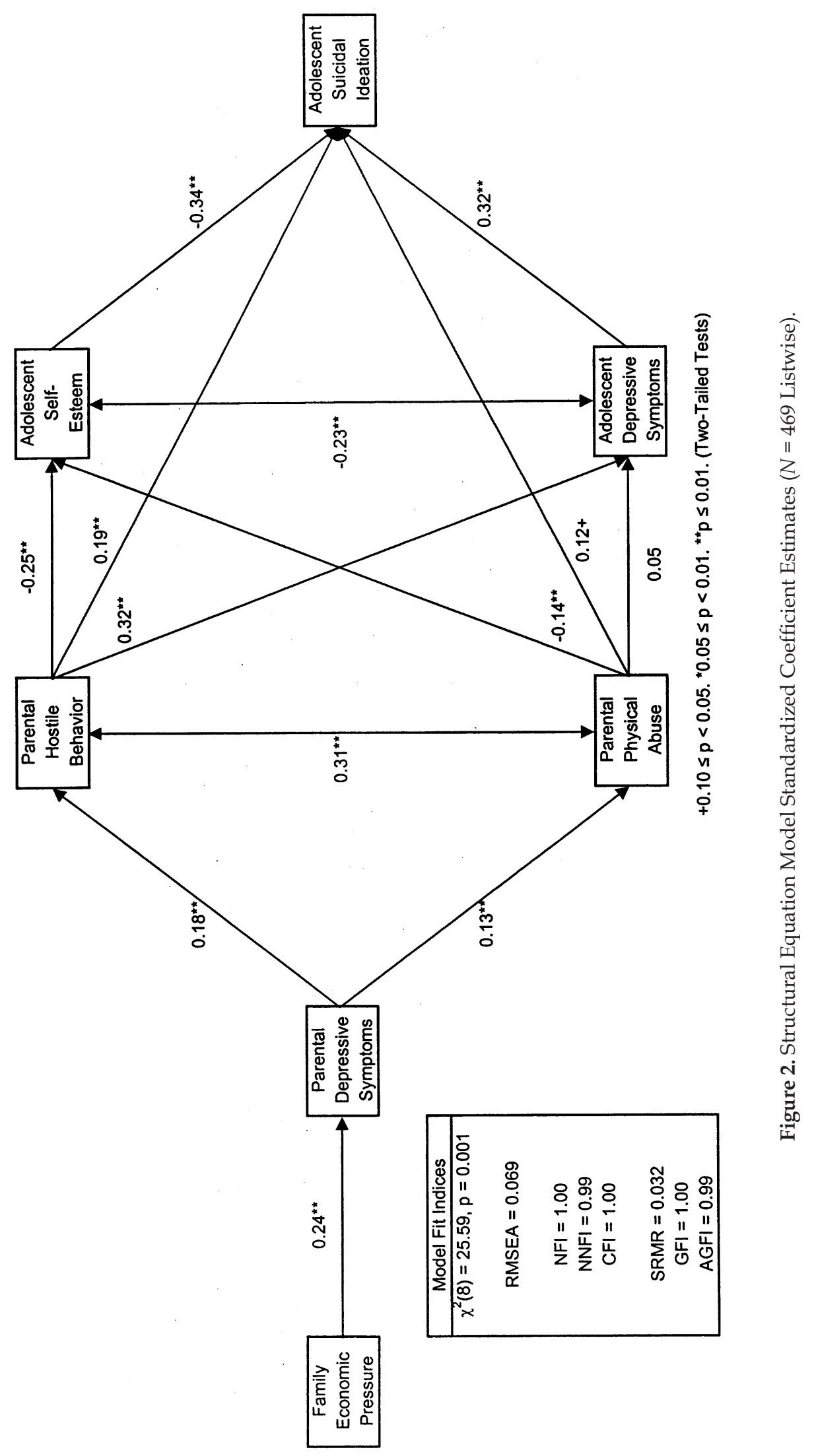


to adolescent depressive symptoms $(\beta=-0.23)$ and suicidal ideation $(\beta=-0.34)$, and adolescent depressive symptoms is positively related to suicidal ideation $(\beta=0.32)$.

\section{Discussion}

This study used a sample of 501 families from the Mississippi Delta region to examine the feasibility of the Family Stress Model (FSM) for understanding the relationships of adolescent suicidal ideation with family economic pressure, parental depressive symptoms, parental hostile behavior and physical abuse, and adolescent self-esteem and depressive symptoms. With the exception of the relationships of parental physical abuse with adolescent depressive symptoms and suicidal ideation, the hypothesized model was supported. Family economic pressure was related to parental depressive symptoms and indirectly related to the remaining variables in the model, which is consistent with other research findings (e.g., Conger et al., 1994).

In addition, parental depressive symptoms was related to parental hostile behavior and physical abuse and indirectly related to adolescent self-esteem, depressive symptoms, and suicidal ideation. Interestingly, parental depressive symptoms was related to adolescent self-esteem and depressive symptoms at the bivariate level, but the multivariate results indicated an indirect relationship via parenting behaviors. The findings in this study are consistent with other studies indicating the important mediating role that parenting behaviors play in the relationship between parental and offspring depression (e.g., Barrera et al., 2002) and between parental depression and adolescent suicidal ideation (Garber et al., 1998).

Moreover, parental hostile behaviors had strong relationships with adolescent self-esteem, depressive symptoms, and suicidal ideation. In fact, the relationships of parental hostile behaviors with adolescent self-esteem and depressive symptoms were among the strongest in the model, and the indirect relationship between parental hostile behav- iors and adolescent suicidal ideation was, by far, the strongest indirect relationship in the model. In contrast, parental physical abuse was not significantly related to adolescent depressive symptoms, was modestly related to adolescent self-esteem, and was moderately indirectly related to adolescent suicidal ideation. That parental hostile behaviors was more important than was parental physical abuse was consistent with the findings of Simons et al. (1994). They found that the quality of parental involvement - but not the use of corporal punishment-was significantly related to adolescent psychological well-being. They suggested that the poor quality of parenting that often accompanies physical punishment (and presumably physical abuse) was generally more important for adolescent psychological functioning than was the punishment itself.

Finally, the finding that both adolescent self-esteem and depressive symptoms were related to adolescent suicidal ideation is consistent with the finding of numerous other studies (e.g., Overholser et al., 1995). This finding, however, conflicts with other studies that failed to find a significant relationship between self-esteem and suicidal ideation when depression is included in the model (e.g., Wild, Flisher, \& Lombard, 2004).

Some weaknesses of this study should be noted. First, although the FSM has been supported by well-designed longitudinal studies (e.g., Conger et al., 1994), the design for the present study was cross-sectional in nature, and thus, a causal interpretation cannot be applied to the findings. It is possible that some of the relationships indicated in the theoretical model operate in the opposite direction or that some of the variables are reciprocally related. For instance, it is possible that adolescent self-esteem or depressive symptoms might influence parental behavior (Conger et al., 1992; Gecas \& Schwalbe, 1986) or the youth's perception of parental behavior. It is also conceivable that parental depressive symptoms could bias parental perceptions of family economic pressure. Clearly, longitudinal data would provide a better test of the applicability of the FSM to adolescent suicidal ideation. 


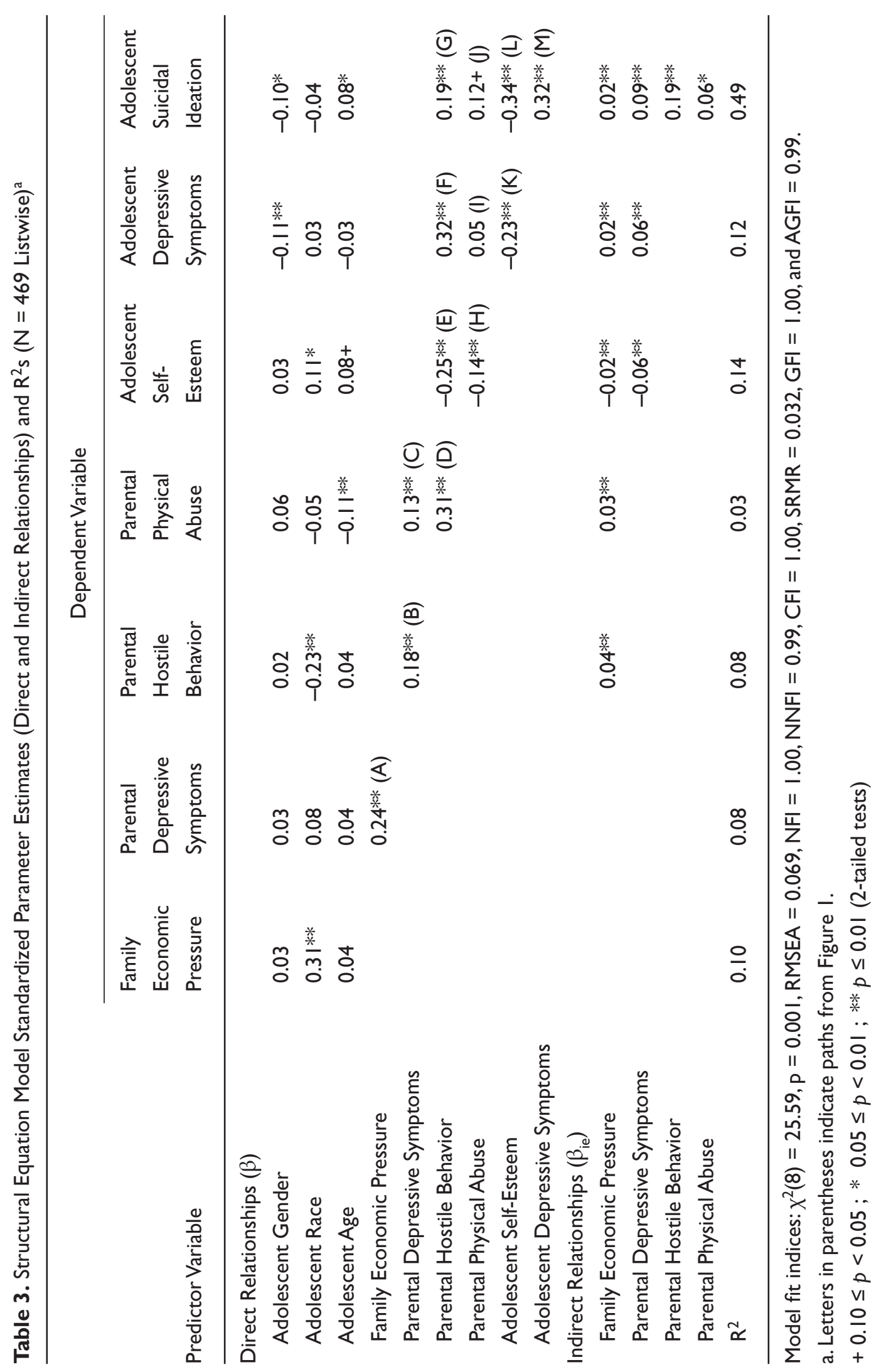


A second weakness involves the sample. Although the families were randomly selected, the inclusion criteria and restricted geographic region make it difficult to generalize the results to other populations. Third, the relationships among variables measured for the same person could have been inflated due to same-reporter bias. Fourth, the model did not include potentially important variables (e.g., hopelessness; Overholser et al., 1995). Fifth, the relationships among parental depressive symptoms, hostile behavior, and physical abuse are potentially problematic because they might not all refer to the same person. Sixth, suicidal ideation was measured with two items that were combined into one variable. This single dichotomous variable could not capture the breadth and variability in suicidal ideation, and the reliability of the variable could not be established. Finally, although the analyses controlled for gender and race, it might have been of interest to determine if any of the relationships in the model differed depending on race and gender. The sample size requirements for structural equation modeling (especially for the Weighted Least Squares procedure) made subgroup analyses impractical in this study.

Despite these weaknesses, the results of the present study were consistent with previous research and provide preliminary evidence for the feasibility of the Family Stress Model to adolescent suicidal ideation. The results highlight the important relationships of adolescent suicidal ideation with family economic pressure, parental depressive symptoms and behaviors, and adolescent selfesteem and depressive symptoms.

\section{References}

Allen, D. M., \& Tarnowski, K. J. (1989). Depressive characteristics of physically abused children. Journal of Abnormal Child Psychology, 17, 1-11.

Allgood-Merten, B., Lewinsohn, P. M., \& Hops, H. (1990). Sex differences and adolescent depression. Journal of Abnormal Psychology, 99, 55-63.

Barrera, M., Prelow, H. M., Dumka, L. E., Gonzales, N. A., Knight, G. P., Michaels, M. L., et al. (2002). Pathways from family economic conditions to ad- olescents' distress: Supportive parenting, stressors outside the family, and deviant peers. Journal of Community Psychology, 30, 135-152.

Beck, A. T. (1976). Cognitive therapy and the emotional disorders. New York: International Universities Press.

Bensley, L. S., Van Eenwyk, J., Spieker, S. J., \& Schoder, J. (1999). Self-reported abuse history and adolescent problem behaviors. I. Antisocial and suicidal behaviors. Journal of Adolescent Health, 24, 163-172.

Bollen, K. A. (1989). Structural equations with latent variables. New York: Wiley.

Chaffin, M., Kelleher, K., \& Hollenberg, J. (1996). Onset of physical abuse and neglect: Psychiatric, substance abuse, and social risk factors from prospective community data. Child Abuse \& Neglect, 20, 191-203.

Conger, R. D., Conger, K. J., Elder, G. H., Lorenz, F. O., Simons, R. L., \& Whitbeck, L. B. (1992). A family process model of economic hardship and adjustment of early adolescent boys. Child Development, 63, 526-541.

Conger, R. D., \& Elder, G. H. (1994). Families in troubled times. New York: Aldine De Gruyter.

Conger, R. D., Ge, X., Elder, G. H., Lorenz, F. O., \& Simons, R. L. (1994). Economic stress, coercive family process, and developmental problems of adolescents. Child Development, 65, 541-561.

Conger, R. D., Lorenz, F. O., Elder, G. H., Melby, J. N., Simons, R. L., \& Conger, K. J. (1991). A process model of family economic pressure and early adolescent alcohol use. Journal of Early Adolescence, 11, 430-449.

Crittenden, P. M. (1998). Dangerous behavior and dangerous contexts: A 35-year perspective on research on the developmental effects of child physical abuse. In P. K. Trickett \& C. J. Schellenbach (eds.), Violence against children in the family and the community (pp. 11-38). Washington, DC: American Psychological Association.

Downey, G., \& Coyne, J. C. (1990). Children of depressed parents: An integrative review. Psychological Bulletin, 108, 50-76.

Dubow, E. F., Kausch, D. F., Blum, M. C., Reed, J., \& Bush, E. (1989). Correlates of suicidal ideation and attempts in a community sample of junior high and high school students. Journal of Clinical Child Psychology, 18, 158-166.

Garber, J., Little, S., Hilsman, R., \& Weaver, K. R. (1998). Family predictors of suicidal symptoms in young adolescents. Journal of Adolescence, 21, 445-457. 
Gecas, V., \& Schwalbe, M. L. (1986). Parental behavior and adolescent self-esteem. Journal of Marriage and the Family, 48, 37-46.

Jöreskog, K. G., \& Sörbom, D. (2001). LISREL 8: User's reference guide. Lincolnwood, IL: Scientific Software International.

Kandel, D. B., Raveis, V. H., \& Davies, M. (1991). Suicidal ideation in adolescence: Depression, substance use, and other risk factors. Journal of Youth $\mathcal{E}$ Adolescence, 20, 289-309.

Kaplan, D. (1989). Model modification in covariance structure analysis: Application of the expected parameter change statistic. Multivariate Behavioral Research, 24, 285-305.

Lewinsohn, P. M., Rohde, P., \& Seeley, J. R. (1996). Adolescent suicidal ideation and attempts: Prevalence, risk factors, and clinical implications. Clinical Psychology Science and Practice, 3, 25-46.

McGruder, B., Lorenz, F. O., Hoyt, D., Ge, X. J., \& Montague, R. (1992). Dimensions of parenting: A technical report. Ames, IA: Iowa State University, Center for Family Research in Rural Mental Health.

Oetting, E. R., Swaim, R. C., Edwards, R. W., \& Beauvais, F. (1989). Indian and Anglo adolescent alcohol use and emotional distress: Path models. American Journal of Drug and Alcohol Abuse, 15, 153-172.

Overholser, J. C., Adams, D. M., Lehnert, K. L., \& Brinkman, M. A. (1995). Self-esteem deficits and suicidal tendencies among adolescents. Journal of the American Academy of Child and Adolescent Psychiatry, 34, 919-928.

Pearlin, L. I. (1989). The sociological study of stress. Journal of Health and Social Behavior, 30, 241-256.

Radloff, L. S. (1977). The CES-D scale: A self-report depression scale for research in the general population. Applied Psychological Measurement, 1, 385-401.

Rosenberg, M. (1965). Society and the adolescent self-image. Princeton, NJ: Princeton University Press.

Rosenberg, M., Schooler, C., \& Schoenbach, C. (1989). Self-esteem and adolescent problems: Modeling reciprocal effects. American Sociological Review, 54, 1004-1018.
Saunders, J. (1992). Demography of the Delta. In A. G. Cosby, M. W. Brackin, T. D. Mason, \& E. R. McCulloch (eds.), A social and economic portrait of the Mississippi Delta. Starkville: Mississippi State University, Social Science Research Center.

Schermelleh-Engel, K., Moosbrugger, H., \& Müller, H. (2003). Evaluating the fit of structural equation models: Tests of significance and descriptive goodnessof-fit measures. Methods of Psychological Research Online, 8(2), 23-74; online http://www.uni-landau. de/ agmunde/mpr/issue20/art2/mpr130_13.pdf (accessed May 20, 2004).

Shagle, S. C., \& Barber, B. K. (1993). Effects of family, marital, and parent-child conflict on adolescent selfderogation and suicidal ideation. Journal of Marriage and the Family, 55, 964-974.

Simons, R. L., Johnson, C., \& Conger, R. D. (1994). Harsh corporal punishment versus quality of parenting involvement as an explanation of adolescent maladjustment. Journal of Marriage and the Family, 56, 591-607.

Straus, M. A. (1979). Measuring intrafamily conflict and violence: The conflict tactics (CT) scales. Journal of Marriage and the Family, 41, 75-88.

Wagner, B. M., Silverman, M. A. C., \& Martin, C. E. (2003). Family factors in youth suicidal behaviors. American Behavioral Scientist, 46, 1171-1191.

Whitbeck, L. B., Simons, R. L., Conger, R. D., Lorenz, F. O., Huck, S., \& Elder, G. H. (1991). Family economic hardship, parental support, and adolescent self-esteem. Social Psychology Quarterly, 54, 353-363.

Wild, L. G., Flisher, A. J., \& Lombard, C. (2004). Suicidal ideation and attempts in adolescents: Associations with depression and six domains of self-esteem. Journal of Adolescence, 27, 611-624.

Yung, Y.-F., \& Bentler, P. M. (1994). Bootstrap-corrected ADF test statistics in covariance structure analysis. British Journal of Mathematical and Statistical Psychology, 47, 63-84. 